\title{
A class of fractional order systems with not instantaneous impulses
}

\author{
Xianmin Zhang \\ School of Mathematics and statistics, Yangtze Normal University, Fuling, Chongqing 408100, China.
}

Communicated by D. Baleanu

\begin{abstract}
This paper is concerned with a kind of fractional order systems with Caputo-Hadamard derivative (of order $\mathrm{q} \in \mathbb{C}$ and $\mathfrak{R}(q) \in(1,2)$ ) and not instantaneous impulses. The obtained result uncovers that there exists a general solution for these impulsive systems, which means that the state trajectory of these impulsive systems is non-unique, and it is expounded by a numerical example. (C)2017 All rights reserved.
\end{abstract}

Keywords: Fractional differential equations, impulsive fractional differential equations, not instantaneous impulses, general solution, state trajectory.

2010 MSC: 34A08, 34A37.

\section{Introduction}

Fractional calculus has gained much attention since it provides a key tool to describe hereditary properties of some materials and processes in many fields of science and engineering, and the general theory of fractional calculus can be referred in $[5,12,15]$, and there are some advances in numerical calculation, controllability, chaos synchronization, etc. for fractional differential equations [4, 11, 20-22].

Impulsive differential equations have been focused since it serves as an important tool to characterize the phenomena in which sudden, discontinuous jumps occur in various fields of science and engineering, and impulsive fractional (partial) differential equations have received much attentions $[1,2,6,8,16-18,23$, 26]. However, impulses are instantaneous impulses in many existing papers about the impulsive models, and it can not describe some processes such as evolution processes in pharmacotherapy. Therefore, the authors in [9] presented a kind of impulsive differential equations with not instantaneous impulses, and the authors in [14] continued studying on differential equations with not instantaneous impulses in a $\mathrm{PC}_{\alpha}$-normed Banach space. Next, the fractional differential equations with not instantaneous impulses were considered in $[13,19]$.

Furthermore, the works in $[7,10]$ developed fractional calculus in frame of Caputo-Hadamard fractional derivative, and Caputo-Hadamard fractional differential equations were studied in [3]. Next, the recent results in [24, 25] discovered that Caputo-Hadamard fractional differential equations with instantaneous impulses have general solution, which uncovered that there may be general solution for CaputoHadamard fractional differential equations with not instantaneous impulses. Therefore, we will try to

Email address: z6x2m@126.com, XianminZhang@126.com (Xianmin Zhang)

doi:10.22436/jnsa.010.08.32 
seek general solution for the following Caputo-Hadamard fractional differential equations with not instantaneous impulses:

$$
\begin{cases}\mathrm{C}-\mathrm{H} \mathrm{D}_{\mathrm{a}^{+}}^{\mathrm{q}} z(\mathrm{t})=\mathrm{f}(\mathrm{t}, z(\mathrm{t})), & \mathrm{t} \in\left(\mathrm{s}_{\mathrm{k}}, \mathrm{t}_{\mathrm{k}+1}\right], \mathrm{k}=0,1, \ldots, \mathrm{N}, \\ z(\mathrm{t})=\mathrm{g}_{\mathrm{k}}(\mathrm{t}, z(\mathrm{t})), & \mathrm{t} \in\left(\mathrm{t}_{\mathrm{k}}, \mathrm{s}_{\mathrm{k}}\right], \mathrm{k}=1,2, \ldots, \mathrm{N}, \\ z(\mathrm{a})=z_{\mathrm{a}}, z^{\prime}(\mathrm{a})=\bar{z}_{\mathrm{a}}, & z_{\mathrm{a}}, \bar{z}_{\mathrm{a}} \in \mathbb{C} .\end{cases}
$$

Here $\mathbf{q} \in \mathbb{C}, \mathfrak{R}(q) \in(1,2)$ and $\mathrm{a}>0, \mathrm{C}-\mathrm{H}_{\mathrm{a}} \mathrm{D}_{\mathrm{a}^{+}}^{\mathrm{q}}$ denotes left-sided Caputo-Hadamard fractional derivative of order $q, f:[a, T] \times \mathbb{C} \rightarrow \mathbb{C}$ is an appropriate continuous function, $g_{k}:\left(t_{k}, s_{k}\right] \times \mathbb{C} \rightarrow \mathbb{C}$ which denote not instantaneous impulses are some appropriate continuous functions, $g_{k}^{\prime}\left(s_{k}, z\left(s_{k}\right)\right)$ exist (here $k=$ $1,2, \ldots, N)$, and $a=t_{0}=s_{0}<t_{1} \leqslant s_{1} \leqslant t_{2} \leqslant \cdots \leqslant t_{N} \leqslant s_{N} \leqslant t_{N+1}=T$.

Next, we will introduce some definitions and conclusions in Section 2, and give the equivalent integral equations for a kind of Caputo-Hadamard fractional differential equations with not instantaneous impulses in Section 3. Finally, an example is provided to illustrate the obtained result.

\section{Preliminaries}

Definition 2.1 ([12, p.110]). Let $0<\mathrm{a}<\mathrm{b}<\infty$ be finite or infinite interval of the half-axis $\mathbb{R}^{+}$. The left-sided Hadamard fractional integral of order $\alpha \in \mathbb{C}$ of function $x(t)$ is defined by

$$
H_{a^{+}}^{\alpha} x(t)=\frac{1}{\Gamma(\alpha)} \int_{a}^{t}\left(\ln \frac{t}{s}\right)^{\alpha-1} x(s) \frac{d s}{s}, \quad(a<t<b),
$$

where $\Gamma(\cdot)$ is the Gamma function.

Definition 2.2 ([10, p.4]). Let $\mathfrak{R}(\alpha) \geqslant 0$ and $n=[\mathfrak{R}(\alpha)]+1, x \in\left\{x:[a, b] \rightarrow \mathbb{C}: \delta^{(n-1)} \chi(t) \in A C[a, b]\right\}$, $0<\mathrm{a}<\mathrm{b}<\infty$. The left-sided Caputo-Hadamard fractional derivatives $\mathrm{C}_{-} \mathrm{H}_{\mathrm{a}^{+}}^{\alpha} \mathrm{x}(\mathrm{t})$ exist everywhere on $[a, b]$ and if $\alpha \notin \mathbb{N}_{0}$,

$$
C-H_{a^{+}}^{\alpha} \chi(t)=\frac{1}{\Gamma(n-\alpha)} \int_{a}^{t}\left(\ln \frac{t}{s}\right)^{n-\alpha-1} \delta^{n} \chi(s) \frac{d s}{s}=H \partial_{a^{+}}^{n-\alpha} \delta^{n} \chi(t),
$$

where differential operator $\delta=t \frac{d}{d t}$ with $\delta^{0} \chi(t)=x(t)$. Moreover, if $\alpha=n \in \mathbb{N}_{0}, c-H_{\mathrm{a}^{+}} \mathrm{D}^{\alpha}(\mathrm{t})=\delta^{\mathrm{n}} \chi(\mathrm{t})$. In particular, $\mathrm{C}-\mathrm{H} \mathrm{D}_{\mathrm{a}^{+}}^{0} \mathrm{x}(\mathrm{t})=\mathrm{x}(\mathrm{t})$.

Lemma $2.3([10, p .5])$. Let $\mathfrak{R}(\alpha)>0, \mathrm{n}=[\mathfrak{R}(\alpha)]+1$, and $x \in \mathrm{C}[\mathrm{a}, \mathrm{b}]$. If $\mathfrak{R}(\alpha) \neq 0$ or $\alpha \in \mathbb{N}$, then

$$
\mathrm{C}-\mathrm{H} \mathrm{D}_{\mathbf{a}^{+}}^{\alpha}\left(\mathrm{H}_{\mathbf{a}^{+}}^{\alpha} \mathrm{x}\right)(\mathrm{t})=x(\mathrm{t}) \text {. }
$$

Lemma 2.4 ([10, p.6]). Let $x \in A C_{\delta}^{n}[a, b]$ or $C_{\delta}^{n}[a, b]$ and $\alpha \in \mathbb{C}$, then

$$
H \partial_{a^{+}}^{\alpha}\left(c-H^{2} D_{a^{+}}^{\alpha} x\right)(t)=x(t)-\sum_{k=0}^{n-1} \frac{\delta^{k} x(a)}{k !}\left(\ln \frac{t}{a}\right)^{k} .
$$

Considering a special case in Corollary 17 in [25], we can draw the following conclusion.

Lemma 2.5. Let $w \in \mathbb{C}, \mathfrak{R}(w) \in(1,2), \sum_{i=1}^{0} y_{i}=0$, and $\xi, \zeta$ are two constants. The impulsive system

$$
\left\{\begin{array}{l}
\mathrm{C}-\mathrm{H} D_{\mathrm{a}^{+}}^{w} x(t)=g(t, x(t)), \quad t \in(a, T], t \neq t_{k}(k=1,2, \ldots, m), \\
\left.\Delta x\right|_{t=t_{k}}=x\left(t_{k}^{+}\right)-x\left(t_{k}^{-}\right)=I_{k}\left(x\left(t_{k}^{-}\right)\right) \in \mathbb{C}, k=1,2, \ldots, m, \\
\left.\Delta x^{\prime}\right|_{t=t_{k}}=x^{\prime}\left(t_{k}^{+}\right)-x^{\prime}\left(t_{k}^{-}\right)=\bar{I}_{k}\left(x\left(t_{k}^{-}\right)\right) \in \mathbb{C}, k=1,2, \ldots, m, \\
x(a)=x_{a} \in \mathbb{C}, x^{\prime}(a)=\bar{x}_{a} \in \mathbb{C}
\end{array}\right.
$$


is equivalent to the integral equation

$$
\begin{aligned}
x(t)= & x_{a}+a \bar{x}_{a} \ln \frac{t}{a}+\sum_{i=1}^{k} I_{i}\left(x\left(t_{i}^{-}\right)\right)+\sum_{i=1}^{k} t_{i} \bar{I}_{i}\left(x\left(t_{i}^{-}\right)\right) \ln \frac{t}{t_{i}}+\frac{1}{\Gamma(w)} \int_{a}^{t}\left(\ln \frac{t}{s}\right)^{w-1} g \frac{d s}{s} \\
& +\sum_{i=1}^{k}\left[\xi I_{i}\left(x\left(t_{i}^{-}\right)\right)+\zeta t_{i} \bar{I}_{i}\left(x\left(t_{i}^{-}\right)\right)\right]\left\{\frac { 1 } { \Gamma ( w ) } \left[\int_{a}^{t_{i}}\left(\ln \frac{t_{i}}{s}\right)^{w-1} g \frac{d s}{s}+\int_{t_{i}}^{t}\left(\ln \frac{t}{s}\right)^{w-1} g \frac{d s}{s}\right.\right. \\
& \left.\left.-\int_{a}^{t}\left(\ln \frac{t}{s}\right)^{w-1} g \frac{d s}{s}\right]+\frac{\ln \frac{t}{t_{i}}}{\Gamma(w-1)} \int_{a}^{t_{i}}\left(\ln \frac{t_{i}}{s}\right)^{w-2} g \frac{d s}{s}\right\}, \text { for } t \in\left(t_{k}, t_{k+1}\right], k=0, \ldots, m,
\end{aligned}
$$

provided that the integral in (2.2) exists, here $g=g(s, x(s))$.

\section{Main results}

For convenience, let $f=f(\tau, z(\tau))$ in this section. Let

$$
z(t)=A_{k}+B_{k} \ln t+z_{a}+a \bar{z}_{a} \ln \frac{t}{a}+\frac{1}{\Gamma(q)} \int_{a}^{t}\left(\ln \frac{t}{\tau}\right)^{q-1} f \frac{d \tau}{\tau}, \text { for } t \in\left(s_{k}, t_{k+1}\right] \subset(0, T],
$$

where $A_{k}$ and $B_{k}(k=0,1, \ldots, N)$ are some constants. In fact, Eq. (3.1) satisfies fractional derivative condition of system (1.1) by Definition 2.2. Substituting (3.1) into system (1.1), we have

$$
z(t)= \begin{cases}A_{k}+B_{k} \ln t+z_{a}+a \bar{z}_{a} \ln \frac{t}{a}+\frac{1}{\Gamma(q)} \int_{a}^{t}\left(\ln \frac{t}{\tau}\right)^{q-1} f \frac{d \tau}{\tau}, & \text { for } t \in\left(s_{k}, t_{k+1}\right], k=0, \ldots, N \\ g_{k}(t, z(t)), & \text { for } t \in\left(t_{k}, s_{k}\right], k=1,2, \ldots, N\end{cases}
$$

Thus, we obtain $A_{0}=0, B_{0}=0$,

$$
\begin{aligned}
A_{k}= & g_{k}\left(s_{k}, z\left(s_{k}\right)\right)-z_{a}+a \bar{z}_{a} \ln a-\frac{1}{\Gamma(q)} \int_{a}^{s_{k}}\left(\ln \frac{s_{k}}{\tau}\right)^{q-1} f \frac{d \tau}{\tau} \\
& -\left[s_{k} g_{k}^{\prime}\left(s_{k}, z\left(s_{k}\right)\right)-\frac{1}{\Gamma(q-1)} \int_{a}^{s_{k}}\left(\ln \frac{s_{k}}{\tau}\right)^{q-2} f \frac{d \tau}{\tau}\right] \ln s_{k},
\end{aligned}
$$

and

$$
B_{k}=s_{k} g_{k}^{\prime}\left(s_{k}, z\left(s_{k}\right)\right)-a \bar{z}_{a}-\frac{1}{\Gamma(q-1)} \int_{a}^{s_{k}}\left(\ln \frac{s_{k}}{\tau}\right)^{q-2} f \frac{d \tau}{\tau}
$$

Therefore,

$$
z(t)=\left\{\begin{array}{l}
z_{a}+a \bar{z}_{a} \ln \frac{t}{a}+\frac{1}{\Gamma(q)} \int_{a}^{t}\left(\ln \frac{t}{\tau}\right)^{q-1} f \frac{d \tau}{\tau}, \quad \text { for } t \in\left(a, t_{1}\right], \\
g_{k}(t, z(t)), \quad \text { for } t \in\left(t_{k}, s_{k}\right], k=1,2, \ldots, N, \\
g_{k}\left(s_{k}, z\left(s_{k}\right)\right)-\frac{1}{\Gamma(q)} \int_{a}^{s_{k}}\left(\ln \frac{s_{k}}{\tau}\right)^{q-1} f \frac{d \tau}{\tau}+\frac{1}{\Gamma(q)} \int_{a}^{t}\left(\ln \frac{t}{\tau}\right)^{q-1} f \frac{d \tau}{\tau} \\
\quad+\left[s_{k} g_{k}^{\prime}\left(s_{k}, z\left(s_{k}\right)\right)-\frac{1}{\Gamma(q-1)} \int_{a}^{s_{k}}\left(\ln \frac{s_{k}}{\tau}\right)^{q-2} f \frac{d \tau}{\tau}\right] \ln \frac{t}{s_{k}} \\
\quad \text { for } t \in\left(s_{k}, t_{k+1}\right], k=1,2, \ldots, N .
\end{array}\right.
$$


It is clear that Eq. (3.2) meets all conditions of system (1.1). Moreover, Eq. (3.2) satisfies a hidden condition

$$
\begin{aligned}
& \begin{array}{l}
\left.\quad \lim _{\left[g_{k}(t, z(t))-z_{a}-a \bar{z}_{a} \ln \frac{t}{a}-\frac{1}{\Gamma(q)}\right.} f_{a}^{t}\left(\ln \frac{t}{\tau}\right)^{q-1} f \frac{d \tau}{\tau}\right]=0 \\
\text { for } t \in\left(t_{k}, s_{k}\right] \text { and all } k \in\{1,2, \ldots, N\} \\
\quad \Leftrightarrow z(t)=z_{a}+a \bar{z}_{a} \ln \frac{t}{a}+\frac{1}{\Gamma(q)} \int_{a}^{t}\left(\ln \frac{t}{\tau}\right)^{q-1} f \frac{d \tau}{\tau}, \text { for } t \in(a, T],
\end{array}
\end{aligned}
$$

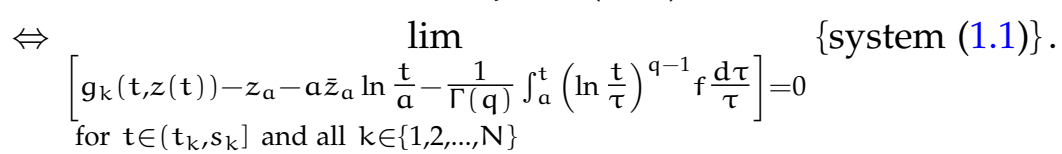

On the other hand, substituting $\tilde{z}(t)=z\left(s_{k}^{+}\right)+\delta z\left(s_{k}^{+}\right) \ln \frac{t}{s_{k}}+\frac{1}{\Gamma(q)} \int_{s_{k}}^{t}\left(\ln \frac{t}{\tau}\right)^{q-1} f \frac{d \tau}{\tau}$ for $t \in\left(s_{k}, t_{k+1}\right]$ (here $\mathrm{k}=0,1, \ldots, \mathrm{N})$ into system $(1.1)$, we have

$$
\hat{z}(t)=\left\{\begin{array}{l}
z_{a}+a \bar{z}_{a} \ln \frac{t}{a}+\frac{1}{\Gamma(q)} \int_{a}^{t}\left(\ln \frac{t}{\tau}\right)^{q-1} f \frac{d \tau}{\tau}, \text { for } t \in\left(a, t_{1}\right], \\
g_{k}(t, z(t)), \quad \text { for } t \in\left(t_{k}, s_{k}\right], k=1,2, \ldots, N \\
g_{k}\left(s_{k}, z\left(s_{k}\right)\right)+s_{k} g_{k}^{\prime}\left(s_{k}, z\left(s_{k}\right)\right) \ln \frac{t}{s_{k}}+\frac{1}{\Gamma(q)} \int_{s_{k}}^{t}\left(\ln \frac{t}{\tau}\right)^{q-1} f \frac{d \tau}{\tau} \\
\quad \text { for } t \in\left(s_{k}, t_{k+1}\right], k=1,2, \ldots, N .
\end{array}\right.
$$

It is clear that $\hat{z}(t)$ satisfies fractional derivative condition and not instantaneous impulses conditions in system (1.1), but it does not satisfy the corresponding hidden condition (3.3). Hence, $\hat{z}(t)$ is only regarded as an approximate solution of system (1.1), and Eq. (3.2) is only a particular solution of system (1.1) since it does not contain $\int_{s_{k}}^{t}\left(\ln \frac{t}{\tau}\right)^{q-1} f \frac{d \tau}{\tau}$, the key part of approximate solution $\hat{z}(t)$ as $t \in\left(s_{k}, t_{k+1}\right.$.

Theorem 3.1. Let $\xi_{k}, \zeta_{k}(k=1,2, \ldots, N)$ are some constants. The system (1.1) is equivalent to an integral equation

$$
z(t)=\left\{\begin{array}{l}
z_{a}+a \bar{z}_{a} \ln \frac{t}{a}+\frac{1}{\Gamma(q)} \int_{a}^{t}\left(\ln \frac{t}{\tau}\right)^{q-1} f \frac{d \tau}{\tau}, \text { for } t \in\left(a, t_{1}\right], \\
g_{k}(t, z(t)), \quad \text { for } t \in\left(t_{k}, s_{k}\right], k=1,2, \ldots, N, \\
\hat{g}_{k}+s_{k} \hat{g}_{k}^{\prime} \ln \frac{t}{s_{k}}+z_{a}+a \bar{z}_{a} \ln \frac{t}{a}+\frac{1}{\Gamma(q)} \int_{a}^{t}\left(\ln \frac{t}{\tau}\right)^{q-1} f \frac{d \tau}{\tau}+\left[\xi_{k} \hat{g}_{k}+\zeta_{k} s_{k} \hat{g}_{k}^{\prime}\right] \\
\cdot\left\{\frac{1}{\Gamma(q)}\left[\int_{a}^{s_{k}}\left(\ln \frac{s_{k}}{\tau}\right)^{q-1} f \frac{d \tau}{\tau}+\int_{s_{k}}^{t}\left(\ln \frac{t}{\tau}\right)^{q-1} f \frac{d \tau}{\tau}-\int_{a}^{t}\left(\ln \frac{t}{\tau}\right)^{q-1} f \frac{d \tau}{\tau}\right]\right. \\
\left.\quad+\frac{\ln \frac{t}{s_{k}}}{\Gamma(q-1)} \int_{a}^{s_{k}}\left(\ln \frac{s_{k}}{\tau}\right)^{q-2} f \frac{d \tau}{\tau}\right\}, \text { for } t \in\left(s_{k}, t_{k+1}\right], k=1,2, \ldots, N,
\end{array}\right.
$$

provided that the integral in (3.4) exists, here $\hat{g}_{k}=g_{k}\left(s_{k}, z\left(s_{k}\right)\right)-z_{a}-a \bar{z}_{a} \ln \frac{s_{k}}{a}-\frac{1}{\Gamma(q)} \int_{a}^{s_{k}}\left(\ln \frac{s_{k}}{\tau}\right)^{q-1} f \frac{d \tau}{\tau}$ and $\hat{g}_{k}^{\prime}=g_{k}^{\prime}\left(s_{k}, x\left(s_{k}\right)\right)-\frac{a \bar{z}_{a}}{s_{k}}-\frac{1}{\Gamma(q-1)} \frac{1}{s_{k}} \int_{a}^{s_{k}}\left(\ln \frac{s_{k}}{\tau}\right)^{q-2} f \frac{d \tau}{\tau}$.

Proof.

Sufficiency. By Lemma 2.4, the solution of system (1.1) as $t \in\left(a, t_{1}\right]$ satisfies

$$
z(t)=z_{a}+a \bar{z}_{a} \ln \frac{t}{a}+\frac{1}{\Gamma(q)} \int_{a}^{t}\left(\ln \frac{t}{\tau}\right)^{q-1} f \frac{d \tau}{\tau}, \text { for } t \in\left(a, t_{1}\right],
$$

and $z(t)=g_{1}(t, z(t))$ for $t \in\left(t_{1}, s_{1}\right]$. 
Next, by above discussion, the approximate solution $\hat{z}(t)$ as $t \in\left(s_{1}, t_{2}\right]$ is given by

$$
\hat{z}(t)=g_{1}\left(s_{1}^{+}, z\left(s_{1}^{+}\right)\right)+s_{1} g_{1}^{\prime}\left(s_{1}^{+}, z\left(s_{1}^{+}\right)\right) \ln \frac{t}{s_{1}}+\frac{1}{\Gamma(q)} \int_{s_{1}}^{t}\left(\ln \frac{t}{\tau}\right)^{q-1} f \frac{d \tau}{\tau}, \text { for } t \in\left(s_{1}, t_{2}\right] .
$$

Let error $e_{1}(t)=z(t)-\hat{z}(t)$ for $t \in\left(s_{1}, t_{2}\right]$, here $z(t)$ denotes the exact solution of system (1.1). Moreover, by the particular solution (3.2), the solution of system (1.1) satisfies

$$
\lim _{\hat{g}_{1} \rightarrow 0, \hat{g}_{1}^{\prime} \rightarrow 0} z(t)=z_{a}+a \bar{z}_{a} \ln \frac{t}{a}+\frac{1}{\Gamma(q)} \int_{a}^{t}\left(\ln \frac{t}{\tau}\right)^{q-1} f \frac{d \tau}{\tau}, \text { for } t \in\left(s_{1}, t_{2}\right],
$$

where $\hat{g}_{1}=g_{1}\left(s_{1}, z\left(s_{1}\right)\right)-z_{a}-a \bar{z}_{a} \ln \frac{s_{1}}{a}-\frac{1}{\Gamma(q)} \int_{a}^{s_{1}}\left(\ln \frac{s_{1}}{\tau}\right)^{q-1} f \frac{d \tau}{\tau}$. Thus,

$$
\begin{aligned}
\lim _{\hat{g}_{1} \rightarrow 0, \hat{g}_{1}^{\prime} \rightarrow 0} e_{1}(t)= & \lim _{\hat{g}_{1} \rightarrow 0, \hat{g}_{1}^{\prime} \rightarrow 0}\{z(t)-\hat{z}(t)\} \\
= & \frac{1}{\Gamma(q)}\left[\int_{a}^{t}\left(\ln \frac{t}{\tau}\right)^{q-1} f \frac{d \tau}{\tau}-\int_{a}^{s_{1}}\left(\ln \frac{s_{1}}{\tau}\right)^{q-1} f \frac{d \tau}{\tau}-\int_{s_{1}}^{t}\left(\ln \frac{t}{\tau}\right)^{q-1} f \frac{d \tau}{\tau}\right] \\
& -\frac{\ln \frac{t}{s_{1}}}{\Gamma(q-1)} \int_{a}^{s_{1}}\left(\ln \frac{s_{1}}{\tau}\right)^{q-2} f \frac{d \tau}{\tau}, \text { for } t \in\left(s_{1}, t_{2}\right] .
\end{aligned}
$$

By (3.5), we make a hypothesis

$$
\begin{aligned}
e_{1}(t)= & \kappa\left(\hat{g}_{1}, \hat{g}_{1}^{\prime}\right) \lim _{\hat{g}_{1} \rightarrow 0, \hat{g}_{1}^{\prime} \rightarrow 0} e_{1}(t) \\
= & \kappa\left(\hat{g}_{1}, \hat{g}_{1}^{\prime}\right)\left\{\frac{1}{\Gamma(q)}\left[\int_{a}^{t}\left(\ln \frac{t}{\tau}\right)^{q-1} f \frac{d \tau}{\tau}-\int_{a}^{s_{1}}\left(\ln \frac{s_{1}}{\tau}\right)^{q-1} f \frac{d \tau}{\tau}-\int_{s_{1}}^{t}\left(\ln \frac{t}{\tau}\right)^{q-1} f \frac{d \tau}{\tau}\right]\right. \\
& \left.-\frac{\ln \frac{t}{s_{1}}}{\Gamma(q-1)} \int_{a}^{s_{1}}\left(\ln \frac{s_{1}}{\tau}\right)^{q-2} f \frac{d \tau}{\tau}\right\}, \text { for } t \in\left(s_{1}, t_{2}\right],
\end{aligned}
$$

where $k(\cdot, \cdot)$ denotes an undetermined function with $\kappa(0,0)=1$. Therefore,

$$
\begin{aligned}
z(t)= & \hat{z}(t)+e_{1}(t) \\
= & g_{1}\left(s_{1}, z\left(s_{1}\right)\right)-\frac{1}{\Gamma(q)} \int_{a}^{s_{1}}\left(\ln \frac{s_{1}}{\tau}\right)^{q-1} f \frac{d \tau}{\tau} \\
& +\left[s_{1} g_{1}^{\prime}\left(s_{1}, z\left(s_{1}\right)\right)-\frac{1}{\Gamma(q-1)} \int_{a}^{s_{1}}\left(\ln \frac{s_{1}}{\tau}\right)^{q-2} f \frac{d \tau}{\tau}\right] \ln \frac{t}{s_{1}}+\frac{1}{\Gamma(q)} \int_{a}^{t}\left(\ln \frac{t}{\tau}\right)^{q-1} f \frac{d \tau}{\tau} \\
& +\left[1-\kappa\left(\hat{g}_{1}, \hat{g}_{1}^{\prime}\right)\right]\left\{\frac{1}{\Gamma(q)}\left[\int_{a}^{s_{1}}\left(\ln \frac{s_{1}}{\tau}\right)^{q-1} f \frac{d \tau}{\tau}+\int_{s_{1}}^{t}\left(\ln \frac{t}{\tau}\right)^{q-1} f \frac{d \tau}{\tau}-\int_{a}^{t}\left(\ln \frac{t}{\tau}\right)^{q-1} f \frac{d \tau}{\tau}\right]\right. \\
& \left.+\frac{\ln \frac{t}{s_{1}}}{\Gamma(q-1)} \int_{a}^{s_{1}}\left(\ln \frac{s_{1}}{\tau}\right)^{q-2} f \frac{d \tau}{\tau}\right\}, \text { for } t \in\left(s_{1}, t_{2}\right] .
\end{aligned}
$$

On the other hand, consider a special case in system (1.1)

$$
\begin{aligned}
& \lim _{t_{1} \rightarrow s_{1}}\left\{\begin{array}{l}
c-H_{a^{+}} D^{q} z(t)=f(t, z(t)), t \in\left(s_{k}, t_{k+1}\right], k=0,1, \\
z(t)=g_{1}(t, z(t)), t \in\left(t_{1}, s_{1}\right], \\
z(a)=z_{a}, z^{\prime}(a)=\bar{z}_{a}, \quad z_{a}, \bar{z}_{a} \in \mathbb{C} .
\end{array}\right. \\
& =\left\{\begin{array}{l}
\mathrm{C}-\mathrm{H}_{\mathrm{a}} \mathrm{D}_{\mathrm{a}^{+}}^{\mathrm{z}} z(\mathrm{t})=\mathrm{f}(\mathrm{t}, z(\mathrm{t})), \mathrm{t} \in\left(\mathrm{s}_{\mathrm{k}}, \mathrm{t}_{\mathrm{k}+1}\right], \mathrm{k}=0,1, \\
z\left(\mathrm{t}_{1}\right)=z\left(\mathrm{~s}_{1}\right)=\mathrm{g}_{1}\left(\mathrm{~s}_{1}, z\left(\mathrm{~s}_{1}\right)\right), \\
z(\mathrm{a})=z_{\mathrm{a}}, z^{\prime}(\mathrm{a})=\bar{z}_{\mathrm{a}}, \quad z_{\mathrm{a}}, \bar{z}_{\mathrm{a}} \in \mathbb{C} .
\end{array}\right.
\end{aligned}
$$




$$
\Leftrightarrow\left\{\begin{array}{l}
\mathrm{C}-\mathrm{H}_{\mathrm{a}^{+}}^{\mathrm{q}} z(\mathrm{t})=\mathrm{f}(\mathrm{t}, z(\mathrm{t})), \mathrm{t} \in\left(\mathrm{s}_{\mathrm{k}}, \mathrm{t}_{\mathrm{k}+1}\right], \mathrm{k}=0,1, \\
z\left(\mathrm{t}_{1}^{+}\right)-z\left(\mathrm{t}_{1}^{-}\right)=\mathrm{g}_{1}\left(\mathrm{~s}_{1}, z\left(\mathrm{~s}_{1}\right)\right)-z_{\mathrm{a}}-\mathrm{a} \bar{z}_{\mathrm{a}} \ln \frac{\mathrm{s}_{1}}{\mathrm{a}}-\frac{1}{\Gamma(\mathrm{q})} \int_{\mathrm{a}}^{\mathrm{s}_{1}}\left(\ln \frac{\mathrm{s}_{1}}{\tau}\right)^{\mathrm{q}-1} \mathrm{f} \frac{\mathrm{d} \tau}{\tau}, \\
z^{\prime}\left(\mathrm{t}_{1}^{+}\right)-z^{\prime}\left(\mathrm{t}_{1}^{-}\right)=g_{1}^{\prime}\left(\mathrm{s}_{1}, z\left(\mathrm{~s}_{1}\right)\right)-\frac{\mathrm{a} \bar{z}_{\mathrm{a}}}{\mathrm{s}_{1}}-\frac{1}{\Gamma(\mathrm{q}-1)} \frac{1}{\mathrm{~s}_{1}} \int_{\mathrm{a}}^{s_{1}}\left(\ln \frac{\mathrm{s}_{1}}{\tau}\right)^{\mathrm{q}-2} \mathrm{f} \frac{\mathrm{d} \tau}{\tau}, \\
z(\mathrm{a})=z_{\mathrm{a}}, z^{\prime}(\mathrm{a})=\bar{z}_{\mathrm{a}}, z_{\mathrm{a}}, \bar{z}_{\mathrm{a}} \in \mathbb{C} .
\end{array}\right.
$$

Using (3.7) and Lemma 2.5 for system (3.8), respectively, we get

$$
\begin{aligned}
1-\kappa\left(\hat{g}_{1}, \hat{g}_{1}^{\prime}\right)= & \xi_{1}\left[g_{1}\left(s_{1}, z\left(s_{1}\right)\right)-z_{a}-a \bar{z}_{a} \ln \frac{s_{1}}{a}-\frac{1}{\Gamma(q)} \int_{a}^{s_{1}}\left(\ln \frac{s_{1}}{\tau}\right)^{q-1} \mathrm{f} \frac{d \tau}{\tau}\right] \\
& +\zeta_{1}\left[s_{1} g_{1}^{\prime}\left(s_{1}, z\left(s_{1}\right)\right)-a \bar{z}_{a}-\frac{1}{\Gamma(q-1)} \int_{a}^{s_{1}}\left(\ln \frac{s_{1}}{\tau}\right)^{q-2} f \frac{d \tau}{\tau}\right]=\xi_{1} \hat{g}_{1}+\zeta_{1} s_{1} \hat{g}_{1}^{\prime} .
\end{aligned}
$$

Thus, Eq. (3.7) can be rewritten as

$$
\begin{aligned}
z(t)= & \hat{g}_{1}+s_{1} \hat{g}_{1}^{\prime} \ln \frac{t}{s_{1}}+z_{a}+a \bar{z}_{a} \ln \frac{t}{a}+\frac{1}{\Gamma(q)} \int_{a}^{t}\left(\ln \frac{t}{\tau}\right)^{q-1} f \frac{d \tau}{\tau}+\left[\xi_{1} \hat{g}_{1}+\zeta_{1} s_{1} \hat{g}_{1}^{\prime}\right] \\
& \cdot\left\{\frac{1}{\Gamma(q)}\left[\int_{a}^{s_{1}}\left(\ln \frac{s_{1}}{\tau}\right)^{q-1} f \frac{d \tau}{\tau}+\int_{s_{1}}^{t}\left(\ln \frac{t}{\tau}\right)^{q-1} f \frac{d \tau}{\tau}-\int_{a}^{t}\left(\ln \frac{t}{\tau}\right)^{q-1} f \frac{d \tau}{\tau}\right]\right. \\
& \left.+\frac{\ln \frac{t}{s_{1}}}{\Gamma(q-1)} \int_{a}^{s_{1}}\left(\ln \frac{s_{1}}{\tau}\right)^{q-2} f \frac{d \tau}{\tau}\right\}, \text { for } t \in\left(s_{1}, t_{2}\right],
\end{aligned}
$$

and $z(t)=g_{2}(t, z(t))$ for $t \in\left(t_{2}, s_{2}\right]$.

Next, the approximate solution $\hat{z}(t)$ for $t \in\left(s_{k}, t_{k+1}\right]$ is provided by

$$
\hat{z}(t)=g_{k}\left(s_{k}^{+}, z\left(s_{k}^{+}\right)\right)+s_{k} g_{k}^{\prime}\left(s_{k}^{+}, z\left(s_{k}^{+}\right)\right) \ln \frac{t}{s_{k}}+\frac{1}{\Gamma(q)} \int_{s_{k}}^{t}\left(\ln \frac{t}{\tau}\right)^{q-1} f \frac{d \tau}{\tau}, \text { for } t \in\left(s_{k}, t_{k+1}\right] .
$$

Let error $e_{k}(t)=z(t)-\hat{z}(t)$ for $t \in\left(s_{k}, t_{k+1}\right]$, and $z(t)$ denotes the exact solution of system (1.1). Furthermore, by the particular solution (3.2), the solution of system (1.1) satisfies

$$
\lim _{\hat{g}_{k} \rightarrow 0, \hat{g}_{k}^{\prime} \rightarrow 0} z(t)=z_{a}+a \bar{z}_{a} \ln \frac{t}{a}+\frac{1}{\Gamma(q)} \int_{a}^{t}\left(\ln \frac{t}{\tau}\right)^{q-1} f \frac{d \tau}{\tau}, \text { for } t \in\left(s_{k}, t_{k+1}\right],
$$

here $\hat{g}_{k}=g_{k}\left(s_{k}, z\left(s_{k}\right)\right)-z_{a}-a \bar{z}_{a} \ln \frac{s_{k}}{a}-\frac{1}{\Gamma(q)} \int_{a}^{s_{k}}\left(\ln \frac{s_{k}}{\tau}\right)^{q-1} f \frac{d \tau}{\tau}$. Therefore,

$$
\begin{aligned}
\lim _{\hat{g}_{k} \rightarrow 0, \hat{g}_{k}^{\prime} \rightarrow 0} e_{k}(t)= & \lim _{\hat{g}_{k} \rightarrow 0, \hat{g}_{k}^{\prime} \rightarrow 0}\{z(t)-\hat{z}(t)\} \\
= & \frac{1}{\Gamma(q)}\left[\int_{a}^{t}\left(\ln \frac{t}{\tau}\right)^{q-1} f \frac{d \tau}{\tau}-\int_{a}^{s_{k}}\left(\ln \frac{s_{k}}{\tau}\right)^{q-1} f \frac{d \tau}{\tau}-\int_{s_{k}}^{t}\left(\ln \frac{t}{\tau}\right)^{q-1} f \frac{d \tau}{\tau}\right] \\
& -\frac{\ln \frac{t}{s_{k}}}{\Gamma(q-1)} \int_{a}^{s_{k}}\left(\ln \frac{s_{k}}{\tau}\right)^{q-2} f \frac{d \tau}{\tau}, \text { for } t \in\left(s_{k}, t_{k+1}\right] .
\end{aligned}
$$

With similarity to (3.6), suppose

$$
\begin{aligned}
e_{k}(t)= & \vartheta\left(\hat{g}_{k}, \hat{g}_{k}^{\prime}\right) \lim _{\hat{g}_{k} \rightarrow 0, \hat{g}_{k}^{\prime} \rightarrow 0} e_{k}(t) \\
= & \vartheta\left(\hat{g}_{k}, \hat{g}_{k}^{\prime}\right)\left\{\frac{1}{\Gamma(q)}\left[\int_{a}^{t}\left(\ln \frac{t}{\tau}\right)^{q-1} f \frac{d \tau}{\tau}-\int_{a}^{s_{k}}\left(\ln \frac{s_{k}}{\tau}\right)^{q-1} f \frac{d \tau}{\tau}-\int_{s_{k}}^{t}\left(\ln \frac{t}{\tau}\right)^{q-1} f \frac{d \tau}{\tau}\right]\right. \\
& \left.-\frac{\ln \frac{t}{s_{k}}}{\Gamma(q-1)} \int_{a}^{s_{k}}\left(\ln \frac{s_{k}}{\tau}\right)^{q-2} f \frac{d \tau}{\tau}\right\}, \text { for } t \in\left(s_{k}, t_{k+1}\right],
\end{aligned}
$$


where $\vartheta(\cdot, \cdot)$ is an undetermined function. Thus,

$$
\begin{aligned}
z(t)= & \hat{z}(t)+e_{k}(t) \\
= & g_{k}\left(s_{k}, z\left(s_{k}\right)\right)-\frac{1}{\Gamma(q)} \int_{a}^{s_{k}}\left(\ln \frac{s_{k}}{\tau}\right)^{q-1} f \frac{d \tau}{\tau} \\
& +\left[s_{k} g_{k}^{\prime}\left(s_{k}, z\left(s_{k}\right)\right)-\frac{1}{\Gamma(q-1)} \int_{a}^{s_{k}}\left(\ln \frac{s_{k}}{\tau}\right)^{q-2} f \frac{d \tau}{\tau}\right] \ln \frac{t}{s_{k}}+\frac{1}{\Gamma(q)} \int_{a}^{t}\left(\ln \frac{t}{\tau}\right)^{q-1} f \frac{d \tau}{\tau} \\
& +\left[1-\vartheta\left(\hat{g}_{k}, \hat{g}_{k}^{\prime}\right)\right]\left\{\frac{1}{\Gamma(q)}\left[\int_{a}^{s_{k}}\left(\ln \frac{s_{k}}{\tau}\right)^{q-1} f \frac{d \tau}{\tau}+\int_{s_{k}}^{t}\left(\ln \frac{t}{\tau}\right)^{q-1} f \frac{d \tau}{\tau}-\int_{a}^{t}\left(\ln \frac{t}{\tau}\right)^{q-1} f \frac{d \tau}{\tau}\right]\right. \\
& \left.+\frac{\ln \frac{t}{s_{k}}}{\Gamma(q-1)} \int_{a}^{s_{k}}\left(\ln \frac{s_{k}}{\tau}\right)^{q-2} f \frac{d \tau}{\tau}\right\}, \text { for } t \in\left(s_{k}, t_{k+1}\right] .
\end{aligned}
$$

Considering a special case of system (1.1), we get

$$
\begin{aligned}
& \lim _{t_{k} \rightarrow s_{k}}\left\{\begin{array}{l}
c-H D_{a^{+}}^{q} z(t)=f(t, z(t)), t \in\left(s_{i}, t_{i+1}\right], i=0,1, \ldots, k, \\
z(t)=z_{a}+a \bar{z}_{a} \ln \frac{t}{a}+\frac{1}{\Gamma(q)} \int_{a}^{t}\left(\ln \frac{t}{\tau}\right)^{q-1} f \frac{d \tau}{\tau}, \text { for } t \in\left(t_{i}, s_{i}\right], i=1,2, \ldots, k-1, \\
z(t)=g_{k}(t, z(t)), t \in\left(t_{k}, s_{k}\right], \\
z(a)=z_{a}, z^{\prime}(a)=\bar{z}_{a}, z_{a}, \bar{z}_{a} \in \mathbb{C} .
\end{array}\right. \\
& =\left\{\begin{array}{l}
\mathrm{C}-\mathrm{H}_{\mathrm{a}^{+}}^{\mathrm{q}} z(\mathrm{t})=f(t, z(t)), \quad t \in\left(s_{i}, t_{i+1}\right], i=0,1, \ldots, k, \\
z(t)=z_{a}+a \bar{z}_{a} \ln \frac{\mathrm{t}}{\mathrm{a}}+\frac{1}{\Gamma(\mathrm{q})} \int_{a}^{t}\left(\ln \frac{\mathrm{t}}{\tau}\right)^{\mathrm{q}-1} \mathrm{f} \frac{\mathrm{d} \tau}{\tau}, \text { for } t \in\left(t_{i}, s_{i}\right], i=1,2, \ldots, k-1, \\
z\left(s_{k}\right)=g_{k}\left(s_{k}, z\left(s_{k}\right)\right), \\
z(a)=z_{a}, z^{\prime}(a)=\bar{z}_{a}, \quad z_{a}, \bar{z}_{a} \in \mathbb{C} .
\end{array}\right.
\end{aligned}
$$

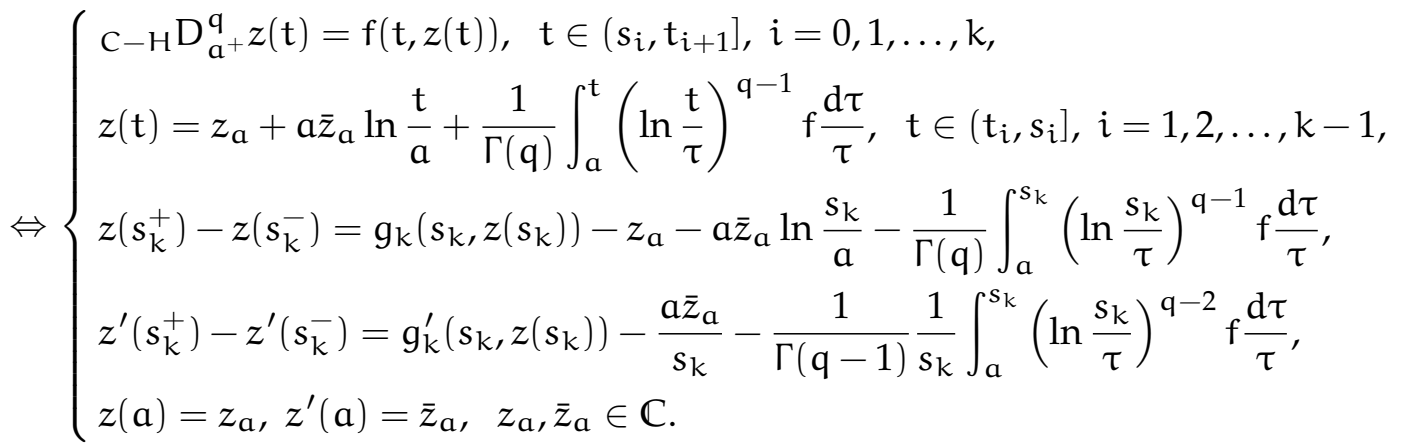

System (3.10) can be regarded as a special case of system (2.1) with single impulsive point. By using (3.9) and Lemma 2.5 for system (3.10), we obtain

$$
\begin{aligned}
1-\kappa\left(\hat{g}_{k}, \hat{g}_{k}^{\prime}\right)= & \xi_{k}\left[g_{k}\left(s_{k}, z\left(s_{k}\right)\right)-z_{a}-a \bar{z}_{a} \ln \frac{s_{k}}{a}-\frac{1}{\Gamma(q)} \int_{a}^{s_{k}}\left(\ln \frac{s_{k}}{\tau}\right)^{q-1} f \frac{d \tau}{\tau}\right] \\
& +\zeta_{k}\left[s_{k} g_{k}^{\prime}\left(s_{k}, z\left(s_{k}\right)\right)-a \bar{z}_{a}-\frac{1}{\Gamma(q-1)} \int_{a}^{s_{k}}\left(\ln \frac{s_{k}}{\tau}\right)^{q-2} f \frac{d \tau}{\tau}\right]=\xi_{k} \hat{g}_{k}+\zeta_{k} s_{k} \hat{g}_{k}^{\prime} .
\end{aligned}
$$

Thus, (3.9) is rewritten as

$$
\begin{aligned}
z(t)= & \hat{g}_{k}+s_{k} \hat{g}_{k}^{\prime} \ln \frac{t}{s_{k}}+z_{a}+a \bar{z}_{a} \ln \frac{t}{a}+\frac{1}{\Gamma(q)} \int_{a}^{t}\left(\ln \frac{t}{\tau}\right)^{q-1} f \frac{d \tau}{\tau}+\left[\xi_{k} \hat{g}_{k}+\zeta_{k} s_{k} \hat{g}_{k}^{\prime}\right] \\
& \cdot\left\{\frac{1}{\Gamma(q)}\left[\int_{a}^{s_{k}}\left(\ln \frac{s_{k}}{\tau}\right)^{q-1} f \frac{d \tau}{\tau}+\int_{s_{k}}^{t}\left(\ln \frac{t}{\tau}\right)^{q-1} f \frac{d \tau}{\tau}-\int_{a}^{t}\left(\ln \frac{t}{\tau}\right)^{q-1} f \frac{d \tau}{\tau}\right]\right.
\end{aligned}
$$




$$
\left.+\frac{\ln \frac{t}{s_{k}}}{\Gamma(q-1)} \int_{a}^{s_{k}}\left(\ln \frac{s_{k}}{\tau}\right)^{q-2} f \frac{d \tau}{\tau}\right\}, \text { for } t \in\left(s_{k}, t_{k+1}\right],
$$

and $z(t)=g_{k+1}(t, z(t))$ for $t \in\left(t_{k+1}, s_{k+2}\right]$.

Necessity. We will verify that Eq. (3.4) satisfies all conditions of system (1.1). Taking the fractional derivative to Eq. (3.4), we have

(i)

$$
\begin{aligned}
\mathrm{C}-\mathrm{H}_{\mathrm{a}^{+}}^{\mathrm{q}}\left[z_{\mathrm{a}}+\mathrm{a} \bar{z}_{\mathrm{a}} \ln \frac{\mathrm{t}}{\mathrm{a}}+\frac{1}{\Gamma(\mathrm{q})} \int_{\mathrm{a}}^{\mathrm{t}}\left(\ln \frac{\mathrm{t}}{\tau}\right)^{\mathrm{q}-1} \mathrm{f} \frac{\mathrm{d} \tau}{\tau}\right] & ={ }_{\mathrm{C}-H} \mathrm{D}_{\mathrm{a}^{+}}^{\mathrm{q}}\left[\frac{1}{\Gamma(\mathrm{q})} \int_{\mathrm{a}}^{\mathrm{t}}\left(\ln \frac{\mathrm{t}}{\tau}\right)^{\mathrm{q}-1} \mathrm{f} \frac{\mathrm{d} \tau}{\tau}\right] \\
& =\mathrm{f}(\mathrm{t}, z(\mathrm{t})), \quad \text { for } \mathrm{t} \in\left(\mathrm{a}, \mathrm{t}_{1}\right],
\end{aligned}
$$

(ii)

$$
\begin{aligned}
c- & D_{a^{+}}^{q}\left\{\hat{g}_{k}+s_{k} \hat{g}_{k}^{\prime} \ln \frac{t}{s_{k}}+z_{a}+a \bar{z}_{a} \ln \frac{t}{a}+\frac{1}{\Gamma(q)} \int_{a}^{t}\left(\ln \frac{t}{\tau}\right)^{q-1} f \frac{d \tau}{\tau}\right. \\
& +\left[\xi_{k} \hat{g}_{k}+\zeta_{k} s_{k} \hat{g}_{k}^{\prime}\right]\left\{\frac{1}{\Gamma(q)}\left[\int_{a}^{s_{k}}\left(\ln \frac{s_{k}}{\tau}\right)^{q-1} f \frac{d \tau}{\tau}+\int_{s_{k}}^{t}\left(\ln \frac{t}{\tau}\right)^{q-1} f \frac{d \tau}{\tau}-\int_{a}^{t}\left(\ln \frac{t}{\tau}\right)^{q-1} f \frac{d \tau}{\tau}\right]\right. \\
& \left.\left.+\frac{\ln \frac{t}{s_{k}}}{\Gamma(q-1)} \int_{a}^{s_{k}}\left(\ln \frac{s_{k}}{\tau}\right)^{q-2} f \frac{d \tau}{\tau}\right\}\right\}_{t \in\left(s_{k}, t_{k+1}\right]} \\
= & \left\{f(t, z(t))_{t \geqslant a}+\frac{1}{\Gamma(q)}\left[\xi_{k} \hat{g}_{k}+\zeta_{k} s_{k} \hat{g}_{k}^{\prime}\right]\right. \\
& \left.\times\left\{c-H D_{s_{k}^{+}}^{q}\left[\int_{s_{k}}^{t}\left(\ln \frac{t}{\tau}\right)^{q-1} f \frac{d \tau}{\tau}\right]-c-H D_{a^{+}}^{q}\left[\int_{a}^{t}\left(\ln \frac{t}{\tau}\right)^{q-1} f \frac{d \tau}{\tau}\right]\right\}\right\}_{t \in\left(s_{k}, t_{k+1}\right]} \\
= & \left\{f(t, z(t))_{t \geqslant a}+\left[\xi_{k} \hat{g}_{k}+\zeta_{k} s_{k} \hat{g}_{k}^{\prime}\right]\left[f(t, z(t))_{t \geqslant s_{k}}-f(t, z(t))_{t \geqslant a}\right]\right\}_{t \in\left(s_{k}, t_{k+1}\right]} \\
= & f(t, z(t))_{t \in\left(s_{k}, t_{k+1}\right] .}
\end{aligned}
$$

Therefore, Eq. (3.4) satisfies $C_{-} \mathrm{H}_{\mathrm{a}^{+}}^{\mathrm{q}} z(t)=f(t, z(t))$ (here $t \in\left(s_{k}, t_{k+1}\right]$ and $k=0,1, \ldots, N$ ) in system (1.1).

Finally, by Eq. (3.4), we have

$$
\begin{aligned}
& \lim _{\left[g_{k}(t, z(t))-z_{a}-a \bar{z}_{a} \ln \frac{t}{a}-\frac{1}{\Gamma(q)} \int^{t}\left(\ln \frac{t}{\tau}\right)^{q-1} f \frac{d \tau}{\tau}\right]=0}\{\text { Eq. (3.4)\} } \\
& \text { for all } k=1,2, \ldots, N \\
& \Leftrightarrow z(\mathrm{t})=z_{\mathrm{a}}+\mathrm{a} \bar{z}_{\mathrm{a}} \ln \frac{\mathrm{t}}{\mathrm{a}}+\frac{1}{\Gamma(\mathrm{q})} \int_{\mathrm{a}}^{\mathrm{t}}\left(\ln \frac{\mathrm{t}}{\tau}\right)^{\mathrm{q}-1} \mathrm{f} \frac{\mathrm{d} \tau}{\tau}, \text { for } \mathrm{t} \in(\mathrm{a}, \mathrm{T}], \\
& \Leftrightarrow\left\{\begin{array}{l}
\mathrm{C}-\mathrm{H}_{\mathrm{a}}^{\mathrm{q}} \mathrm{z}(\mathrm{t})=\mathrm{f}(\mathrm{t}, z(\mathrm{t})), \mathrm{t} \in(\mathrm{a}, \mathrm{T}], \\
z(\mathrm{a})=z_{\mathrm{a}}, z^{\prime}(\mathrm{a})=\bar{z}_{\mathrm{a}}, \quad z_{\mathrm{a}}, \bar{z}_{\mathrm{a}} \in \mathbb{C} .
\end{array}\right.
\end{aligned}
$$

Therefore,

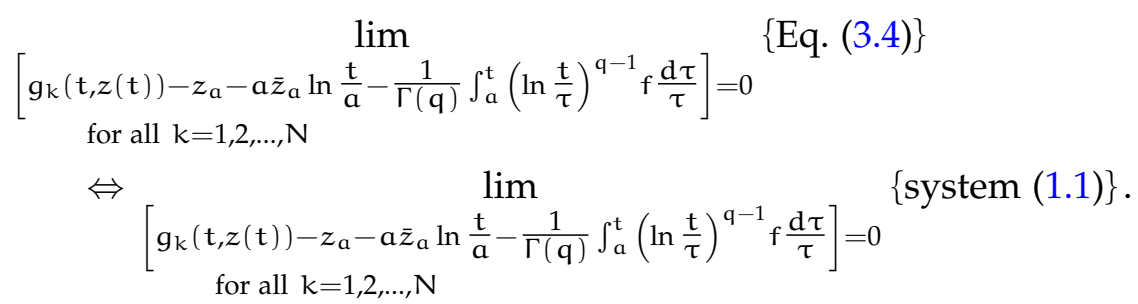

Thus, Eq. (3.4) satisfies all conditions of system (1.1).

According to above proof, the conclusion can be drawn. The proof is now completed. 


\section{An example}

In this section, we will give an example to illustrate that there exists a general solution for the CaputoHadamard fractional differential equations with not instantaneous impulses.

Example 4.1. Let us consider the following impulsive system

$$
\begin{cases}\mathrm{C}-\mathrm{H}^{\frac{3}{2}} \mathrm{1^{+ }} z(\mathrm{t})=\ln \mathrm{t}, & \mathrm{t} \in(1,2] \cup(3,4] \\ z(\mathrm{t})=\mathrm{t}^{2}, & \mathrm{t} \in(2,3] \\ z(1)=1, & z^{\prime}(1)=1\end{cases}
$$

By Theorem 3.1, the general solution of (4.1) can be obtained as

$$
z(t)=\left\{\begin{array}{l}
\left.\begin{array}{l}
1+\ln t+\frac{1}{\Gamma\left(\frac{7}{2}\right)}(\ln t)^{\frac{5}{2}}, \text { for } t \in(1,2], \\
t^{2}, \quad \text { for } t \in(2,3], \\
9-\frac{1}{\Gamma\left(\frac{7}{2}\right)}(\ln 3)^{\frac{5}{2}}+\left.\left[18-\frac{1}{\Gamma\left(\frac{5}{2}\right)}(\ln 3)^{\frac{3}{2}}\right] \ln \frac{t}{3}\right|_{t \geqslant 3}+\left.\frac{1}{\Gamma\left(\frac{7}{2}\right)}(\ln t)^{\frac{5}{2}}\right|_{t>1} \\
\quad+\left\{\xi\left[8-\ln 3-\frac{1}{\Gamma\left(\frac{7}{2}\right)}(\ln 3)^{\frac{5}{2}}\right]+\zeta\left[17-\frac{1}{\Gamma\left(\frac{5}{2}\right)}(\ln 3)^{\frac{3}{2}}\right]\right\} \\
\quad\left\{\frac{1}{\Gamma\left(\frac{7}{2}\right)}\left[(\ln 3)^{\frac{5}{2}}+\left(\ln \frac{t}{3}\right)^{\frac{3}{2}}\left[\ln t+\frac{3 \ln 3}{2}\right]_{t \geqslant 3}-\left.(\ln t)^{\frac{5}{2}}\right|_{t>1}\right]+\left.\frac{(\ln 3)^{\frac{3}{2}}}{\Gamma\left(\frac{5}{2}\right)} \ln \frac{t}{3}\right|_{t \geqslant 3}\right.
\end{array}\right\}
\end{array}\right.
$$

here $\xi$ and $\zeta$ are two constants. To show solution trajectory of system (4.1), we plot (4.2) for several values of $\xi$ and $\zeta$ in Fig. 1.

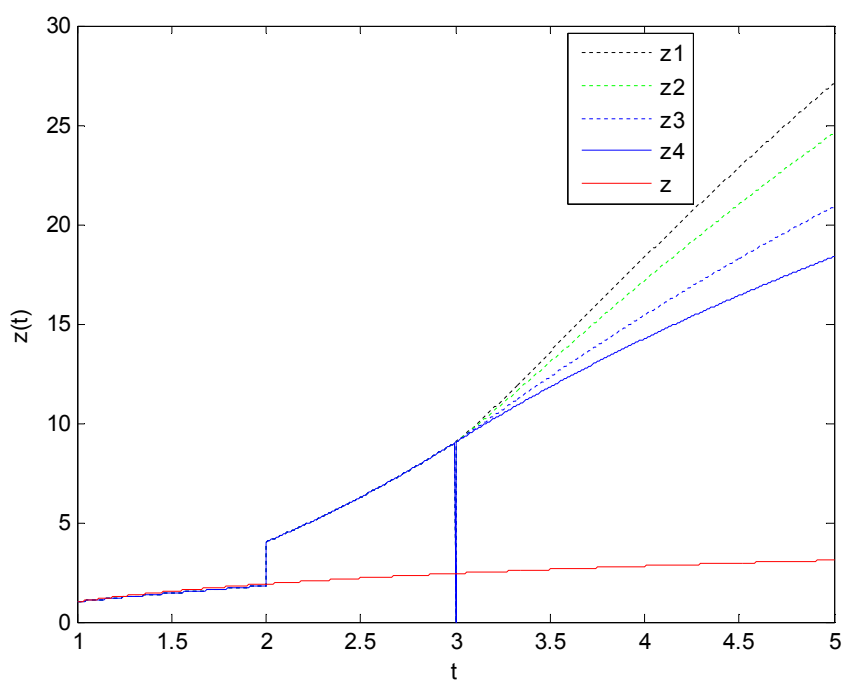

Figure 1: Solution trajectory of system (4.1).

$z 1, z 2, z 3$, and $z 4$ denote (4.2) with $\xi=2$ and $\zeta=2, \xi=0$ and $\zeta=2, \xi=2$ and $\zeta=0$, and $\xi=0$ and $\zeta=0$, respectively, and $z$ is the solution trajectory of system (4.1) without impulses in Fig. 1 . 


\section{Acknowledgment}

The author is deeply grateful to the anonymous referees for their kind comments, correcting errors and improving written language, which have been very useful for improving the quality of this paper. The work described in this paper is financially supported by the National Natural Science Foundation of China (Grant No. 21576033, 21636004).

\section{References}

[1] S. Abbas, M. Benchohra, Impulsive partial hyperbolic functional differential equations of fractional order with statedependent delay, Fract. Calc. Appl. Anal., 13 (2010), 225-242. 1

[2] S. Abbas, M. Benchohra, Upper and lower solutions method for impulsive partial hyperbolic differential equations with fractional order, Nonlinear Anal. Hybrid Syst., 4 (2010), 406-413. 1

[3] Y. Adjabi, F. Jarad, D. Baleanu, T. Abdeljawad, On Cauchy problems with Caputo Hadamard fractional derivatives, J. Comput. Anal. Appl., 21 (2016), 661-681. 1

[4] Y.-R. Bai, Hadamard fractional calculus for interval-valued functions, J. Comput. Complex. Appl., 3 (2017), 23-43. 1

[5] D. Baleanu, K. Diethelm, E. Scalas, J. J. Trujillo, Fractional calculus, Models and numerical methods, Series on Complexity, Nonlinearity and Chaos, World Scientific Publishing Co. Pte. Ltd., Hackensack, NJ, (2012). 1

[6] J.-X. Cao, H.-B. Chen, Some results on impulsive boundary value problem for fractional differential inclusions, Electron. J. Qual. Theory Differ. Equ., 2011 (2011), 24 pages. 1

[7] Y. Y. Gambo, F. Jarad, D. Baleanu, T. Abdeljawad, On Caputo modification of the Hadamard fractional derivatives, Adv. Difference Equ., 2014 (2014), 12 pages. 1

[8] T.-L. Guo, K.-J. Zhang, Impulsive fractional partial differential equations, Appl. Math. Comput., 257 (2015), 581-590. 1

[9] E. Hernández, D. O’Regan, On a new class of abstract impulsive differential equations, Proc. Amer. Math. Soc., 141 (2013), 1641-1649. 1

[10] F. Jarad, T. Abdeljawad, D. Baleanu, Caputo-type modification of the Hadamard fractional derivatives, Adv. Difference Equ., 2012 (2012), 8 pages. 1, 2.2, 2.3, 2.4

[11] S. Kailasavalli, D. Baleanu, S. Suganya, M. M. Arjunan, Exact controllability of fractional neutral integro-differential systems with state-dependent delay in Banach spaces, An. Ştiinţ. Univ. "Ovidius" Constana Ser. Mat., 24 (2016), $29-55$. 1

[12] A. A. Kilbas, H. H. Srivastava, J. J. Trujillo, Theory and applications of fractional differential equations, North-Holland Mathematics Studies, Elsevier Science B.V., Amsterdam, (2006). 1, 2.1

[13] P.-L. Li, C.-J. Xu, Mild solution of fractional order differential equations with not instantaneous impulses, Open Math., 13 (2015), 436-443. 1

[14] M. Pierri, D. O'Regan, V. Rolnik, Existence of solutions for semi-linear abstract differential equations with not instantaneous impulses, Appl. Math. Comput., 219 (2013), 6743-6749. 1

[15] I. Podlubny, Fractional differential equations, An introduction to fractional derivatives, fractional differential equations, to methods of their solution and some of their applications, Mathematics in Science and Engineering, Academic Press, Inc., San Diego, CA, (1999). 1

[16] I. Stamova, G. Stamov, Stability analysis of impulsive functional systems of fractional order, Commun. Nonlinear Sci. Numer. Simul., 19 (2014), 702-709. 1

[17] Y.-S. Tian, Z.-B. Bai, Existence results for the three-point impulsive boundary value problem involving fractional differential equations, Comput. Math. Appl., 59 (2010), 2601-2609.

[18] J.-R. Wang, Y.-R. Zhang, On the concept and existence of solutions for fractional impulsive systems with Hadamard derivatives, Appl. Math. Lett., 39 (2015), 85-90. 1

[19] J.-R. Wang, Y. Zhou, Z. Lin, On a new class of impulsive fractional differential equations, Appl. Math. Comput., 242 (2014), 649-657. 1

[20] G.-C. Wu, D. Baleanu, Chaos synchronization of the discrete fractional logistic map, Signal Process., 102 (2014), 96-99. 1

[21] G.-C. Wu, D. Baleanu, Discrete fractional logistic map and its chaos, Nonlinear Dynam., 75 (2014), $283-287$.

[22] G.-C. Wu, D. Baleanu, H.-P. Xie, F.-L. Chen, Chaos synchronization of fractional chaotic maps based on the stability condition, Phys. A, 460 (2016), 374-383. 1

[23] X.-M. Zhang, On the concept of general solution for impulsive differential equations of fractional-order $\mathrm{q} \in(1,2)$, Appl. Math. Comput., 268 (2015), 103-120. 1

[24] X.-M. Zhang, The general solution of differential equations with Caputo-Hadamard fractional derivatives and impulsive effect, Adv. Difference Equ., 2015 (2015), 16 pages. 1

[25] X.-M. Zhang, X.-Z. Zhang, Z.-H. Liu, H. Peng, T. Shu, S.-Y. Yang, The general solution of impulsive systems with Caputo-Hadamard fractional derivative of order $q \in \mathbb{C}(\operatorname{Re}(q) \in(1,2))$, Math. Probl. Eng., 2016 (2016), 20 pages. 1, 2

[26] X.-M. Zhang, X.-Z. Zhang, M. Zhang, On the concept of general solution for impulsive differential equations of fractional order $\mathrm{q} \in(0,1)$, Appl. Math. Comput., 247 (2014), 72-89. 1 